\title{
Obstrução da via de saída de ventrículo esquerdo por prótese mitral: apresentação de seis casos
}

\author{
Pablo M. A. POMERANTZEFF*, Yukio KIOKA*, Lauro T. KAWABE*, José Otávio C. AULER Jr.", Edgard
} A. LOPES*, Geraldo VERGINELLI*, Adib D. JATENE*

RBCCV 44205-115

POMERANTZEFF, P. M. A.; KIOKA, Y.; KAWABE, L. T.; AULER Jr., J. O. C.; LOPES, E. A.; VERGINELLI, G.; JATENE, A. D. - Obstrução da via de saída de ventrículo esquerdo por prótese mitral: relato de seis casos. Rev. Bras. Cir. Cardiovasc., 5(2): 120-124, 1990.

RESUMO: De janeiro de 1982 a março de 1984, foram implantadas 170 biopróteses de pericárdio bovino e alto perfil, no Instituto do Coração do Hospital das Clínicas da Faculdade de Medicina da Universidade de São Paulo, com mortalidade hospitalar de 7,1\%. Através do estudo anatomopatológico, foram identificados cinco casos em que ocorreu obstrução da via de saída do ventrículo esquerdo após substituiçāo da valva mitral. Com base nessa experiência, a indicação de estudo hemodinâmico no 1 : dia de pós-operatório em paciente em baixo débito, sem explicação, possibilitou identificar obstrução da via de saída do ventrículo esquerdo, com retroca valvar e boa evoluçāo. Neste trabalho, sāo relatados detalhadamente os seis casos e discutidas as causas da obstrução da via de saída na substituição mitral.

DESCRITORES: ventrículo esquerdo, via de saída, obstrução; próteses valvulares cardíacas, cirurgia, complicaçōes; valva mitral, cirurgia, complicaçōes.

\section{INTRODUÇĀO}

A primeira troca de valva mitral foi realizada por STARR \& EDWARDS ${ }^{8}$, em 1961, sendo implantada uma prótese de bola. Após isso, várias próteses mecânicas e biológicas foram desenvolvidas, sendo também aprimoradas as técnicas de proteção miocárdica e tratamento pós-operatório. Dependendo do tipo de prótese utilizada, podem ocorrer problemas no pós-operatório imediato, como, por exemplo, trombose aguda ou mau funcionamento do elemento oclusor em prótese mecânica, insuficiência miocárdica por desproporção da prótese que foi implantada, ou, ainda, a possibilidade de obstrução de via de saída de ventrículo esquerdo (OVSVE) tem sido descrita na literatura ${ }^{1,6}$. Em nosso meio, esta complicação é raramente documentada, os óbitos sendo rotulados como devido a baixo débitos, insuficiência miocárdica, ou sem causa aparente.
Neste trabalho, são relatados seis casos de obstrução da via de saída do ventrículo esquerdo (OVSVE), sendo cinco deles identificados através de estudo necroscópico que, em nossa instituição, é realizado de rotina.

\section{CASUÍSTICA E MÉTODOS}

Entre janeiro de 1982 e março de 1984, foram implantadas, no InCor-HC-FMUSP, 170 biopróteses de pericárdo bovino confeccionadas com anel flexível de polipropileno de alto perfil. A mortalidade hospitalar foi de 12 pacientes $(7,1 \%)$, sendo que, em cinco deles, foi identificada OVSVE no estudo anatomopatológico. Com base nesta experiência, a indicação de estudo hemodinâmico no 1 : dia de pós-operatório em paciente com baixo débito sem explicação permitiu identificar OVSVE.

\footnotetext{
Trabalho realizado no Instituto do Coração do Hospital das Clínicas da Faculdade de Medicina da Universidade de São Paulo. São Paulo, SP, Brasil. Recebido para publicaçăo em 1: de fevereiro de 1990.

* Do Instituto do Coração do Hospital das Clinicas da Faculdade de Medicina da Universidade de São Paulo.

Endereço para separatas: Pablo Pomerantzeff. Av. Dr. Enéas Carvalho de Aguiar, 44. Divisăo Cirúrgica. 05403 São Paulo, SP, Brasil.
} 
POMERANTZEFF, P. M. A.; KIOKA, Y.; KAWABE, L. T.; AULER Jr., J. O. C.; LOPES, E. A.; VeRGINELLI, G.; JATENE, A. D. - Obstrução da via de saída de ventrículo esquerdo por prótese mitral: relato de seis casos. Rev. Bras. Cir. Cardiovasc., 5(2): 120-124, 1990.

CASO 1 - F. A. S., masculino, raça branca, 19 anos, com história de correção cirúrgica de drenagem anômala parcial de veias pulmonares e plástica de De Vega em valva tricúspide aos 16 anos de idade, e história atual de dispnéia progressiva, febre e anemia, tendo sido diagnosticada encodardite bacteriana. Apesar do tratamento clínico, o paciente evoluiu para insuficiência cardiaca classe funcional (CF) IV da New York Heart Association (NYHA). Ao exame físico no dia da operação, o paciente encontrava-se em mau estado geral, com ortopnéia e afebril. $\mathrm{Na}$ ausculta pulmonar, apresentava estertores subcrepitantes nas bases, e na ausculta cardíaca sopro sistólico (SS) $++/ 3$ em área mitral e SS +/3 em área tricúspide. O fígado era percutível e palpável $\mathrm{a} \pm 2 \mathrm{~cm}$ do rebordo costal direito $(R C D)$. O eletrocardiograma (ECG) mostrava ritmo ectópico atrial, sobrecarga atrial esquerda e sobrecarga biventricular. $\mathrm{O}$ ecocardiograma (ECO) mostrava insuficiência mitral de grau moderado com vegetaçōes na valva mitral. O cateterismo cardíaco demonstrou regurgitação mitral $+++1++++$, com hipertensāo moderada em câmaras direitas e elevação das pressōes de capilar pulmonar (CP) e diastólica final de ventrículo esquerdo ( $\left.\mathrm{Pd}_{2} \mathrm{VE}\right)$. No dia 09.03.1982, foi feita a substituição da valva mitral por bioprótese de pericárdio bovino de alto perfil (PBAP) n: 26, montada em anel flexível de polipropileno, e substituição da valva tricúspide por PBAP n: 28.

$\mathrm{Na}$ sala de operações, o paciente necessitou de drogas inotrópicas, evoluindo em baixo débito no pós-operatório imediato, retornando à sala de operações após hipotensão severa, porém sem recuperação possivel.

A necropsia mostrou hipertrofia e dilatação dos ventrículos, sinais de congestão passiva crônica pulmonar e hepática e bioprótese mitral obstruindo a via de saída do ventrículo esquerdo.

CASO 2 - J. B. S. R., masculino, raça branca, 24 anos, tendo sido submetido a comissurotomia mitral aos 10 anos de idade. Voltou a apresentar sintomatologia aos 18 anos de idade, com piora progressiva, evoluindo para CF IV há um ano.

O paciente foi internado em mau estado geral, em anasarca e com ascite, no dia 15.02.1982; apresentou melhora após tratamento clínico.

Ao exame físico, no dia 01.03.1982, pesava $47 \mathrm{~kg}$, com mucosas descoradas $+/ 4$, acianótico e eupnéico, em repouso. A freqüência cardiaca (FC) era de 96 batimentos por minuto, a freqüência respiratória de 26 e a pressão arterial (PA) de $110 \times 70 \mathrm{mmHg}$.

Havia diminuiçāo do murmúrio vesicular em ambas as bases pulmonares. $O$ ictus era palpado no $7^{\circ}$. espaço intercostal esquerdo (EIE) para fora da linha hemiclavicular (LHC). Na ausculta cardiaca, apresentava SS $+++/ 3$ e sopro diastólico (SD) $+/ 3$ em foco mitral; $\mathrm{SS}+/ 3$ em foco tricúspide. Abdome globoso, ascite $+/ 3$ e fígado a $6 \mathrm{~cm}$ de RCD. O ECG apresentava fibrilação atrial, bloqueio atrioventricular variável e distúrbio de condução de ramo direito. O diagnóstico pelo ECO foi de dupla lesão mitral e insuficiência tricúspide com átrio esquerdo (AE) medindo $105 \mathrm{~mm}$ e diâmetro diastólico de VE (DDVE) de $78 \mathrm{~mm}$. O cateterismo cardíaco demonstrava dupla lesão mitral, insuficiência aórtica discreta e hipertensão pulmonar.

Em 15.03.1982, foi realizada a substituição da valva mitral por bioprótese de PBAP n: 26 e plástica de De Vega na tricúspide. $\mathrm{O}$ paciente evoluiu com quadro de insuficiência cardiaca com óbito no 61 dia de pós-operatório.

O estudo anatomopatológico demonstrou congestão passiva crônica nos pulmōes e o coração com enorme dilatação do átrio esquerdo com trombos murais, ventrículo esquerdo com discreta hipertrofia e dilatação, e via de saída de ventrículo esquerdo parcialmente obstruída pela prótese mitral.

CASO 3 - E. F. L. M., feminina, raça negra, 25 anos, com história de cansaço e dispnéia aos esforços desde aos seis anos de idade, controlada clinicamente. Aos 25 anos, apresentou piora do quadro de dispnéia e edema de membros inferiores, com progressão da insuficiência cardiaca para CF IV. Ao exame físico, apresentava SS $+++/ 3$ em área mitral, fígado a $4 \mathrm{~cm}$ do RCD e edema de membros inferiores. O ECG mostrava sobrecarga ventricular esquerda e alteraçōes da repolarização ventricular. A conclusão do ECO foi de insuficiência mitral com AE medindo $56 \mathrm{~mm}$ e DDVE $56 \mathrm{~mm}$. O estudo hemodinâmico demonstrava insuficiência mitral importante e hipertensão pulmonar discreta.

No dia 08.11.1982, foi realizada troca de valva mitral por PBAP n: 26. No pós-operatório imediato, apresentou síndrome de baixo débito, apesar da administração de inotrópicos, evoluindo com oligúria e insuficiência respiratória. No 3 ? dia de pós-operatório, apresentou bradi-

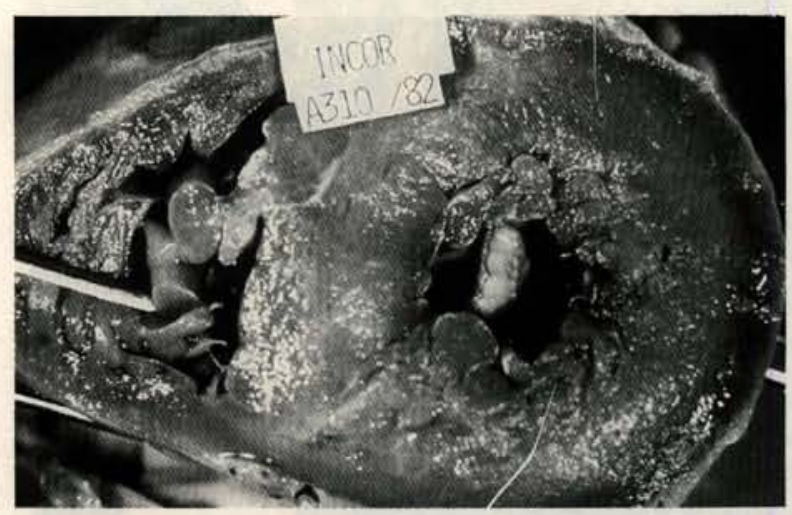

Fig. 1 - Caso 3: Importante hipertrofia do ventrículo esquerdo com a haste da prótese obstruindo a via de saida do ventriculo esquerdo. 
POMERANTZEFF, P. M. A.; KIOKA, Y.; KAWABE, L. T.; AULER Jr., J. O. C.; LOPES, E. A.; VERGINELLI, G.; JATENE, A. D. - Obstrução da via de saída de ventriculo esquerdo por prótese mitral: relato de seis casos. Rev. Bras. Cir. Cardiovasc., 5(2): $120-124,1990$.

cardia e hipotensão severa, seguidas de parada cardiorrespiratória.

O exame anatomopatológico conclui tratar-se de anomalia congênita rara, sendo que alguns dados sugeriam persistência do canal atrioventricular, quais sejam, septo muscular curto, insuficiência mitral com cordas tendíneas curtas inseridas na borda superior do septo muscular, anéis das valvas mitral e tricúspide na mesma altura e a posição da bioprótese bastante obliqua. Havia importante hipertrofia do ventrículo esquerdo e a prótese mitral, situada obliquamente, obstruia a via de saída do ventrículo esquerdo (Figura 1).

CASO 4 - E. L. C., feminina, raça branca, 62 anos, com história de febre reumática, tendo sido submetida a comissurotomia mitral aos 44 anos de idade. Evoluiu assintomática até aos 58 anos de idade, quando se iniciou quadro de dispnéia e cansaço aos médios esforços, diagnosticando-se, nessa época, re-estenose mitral, estando em CF III.

Ao exame físico, apresentava $\mathrm{SD}+++/ 3 \mathrm{em}$ área mitral e $\mathrm{SS}+/ 3$ em área tricúspide, fígado a $8 \mathrm{~cm}$ do

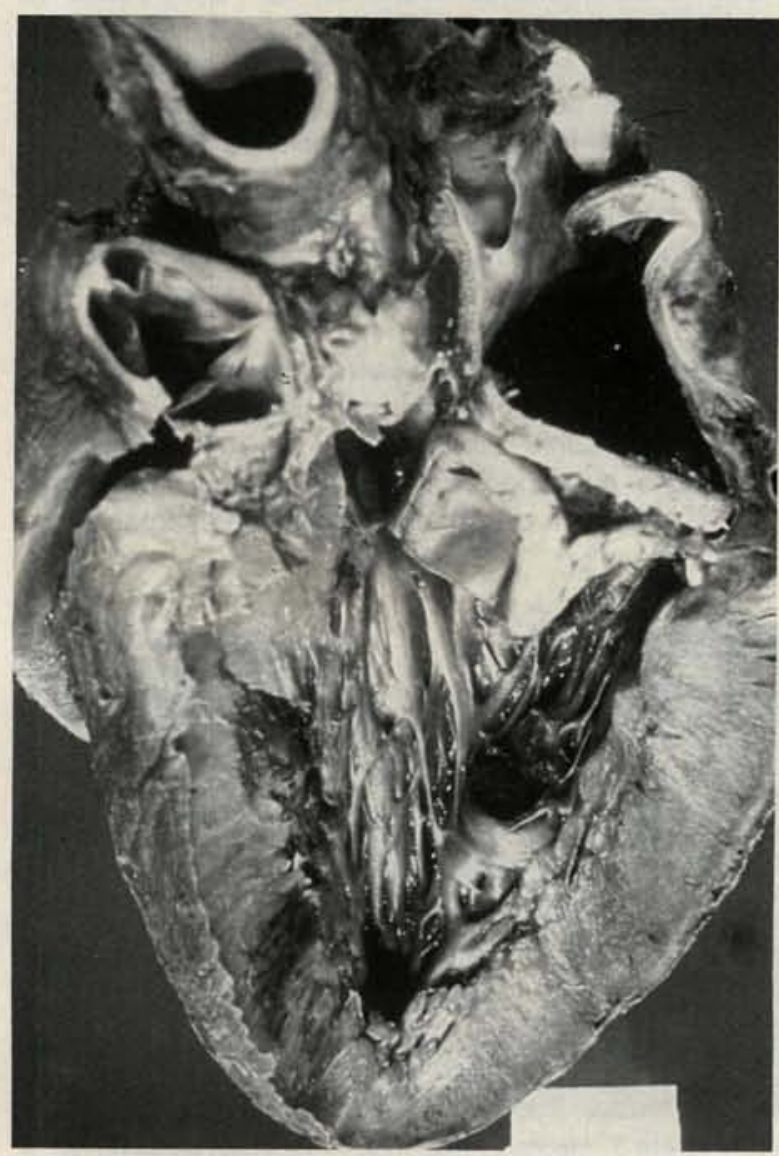

Fig. 2 - Caso 4: Obstruçâo da via de saida do ventrículo esquerdo pela bioprótese com traumatismo do septo interventricular.
RCD, sendo que o ECG mostrava fibrilação atrial, sobrecarga ventricular esquerda, extra-sístoles ventriculares e alteraçōes da repolarizaçāo ventricular. O resultado do ECO foi de estenose mitral com calcificação severa, hipertensão pulmonar, átrio esquerdo de $70 \mathrm{~mm}$ e DDVE de $46 \mathrm{~mm}$. O estudo hemodinâmico demonstrou estenose mitral e hipertensāo pulmonar. A paciente foi submetida a troca de valva mitral em 08.12 .82 , sendo colocada uma bioprótese PBAP n: 24. No pós-operatório imediato, apresentou hemiparesia esquerda e convulsão, evoluindo com baixo débito não muito acentuado. No 8 : dia de pós-operatório, apresentou dispnéia e arritmia ventricular, seguindo-se hipotensăo severa, com evoluçāo para óbito.

O estudo anatomopatológico revelou hipertrofia do ventrículo esquerdo, dilatação do átrio esquerdo, alguns pontos de infarto subendocárdico com cerca de uma semana de evolução e, macroscopicamente, existência de obstrução da via de saida do ventrículo esquerdo pela bioprótese, com traumatismo do septo interventricular pela haste do anel da bioprótese.

CASO 5 - O. B., feminina, raça branca, 41 anos, com história de febre reumática na infância, tendo sido submetida a comissurotomia mitral aos 20 anos de idade por estenose mitral. Evoluiu assintomática até os 39 anos de idade, quando começou a apresentar dispnéia, progredindo para CF III. Ao exame físico, apresentava, em área mitral, $\mathrm{SS}++/ 3, \mathrm{SD}+++/ 3 \mathrm{e}$, em área tricúspide, $\mathrm{SS}++$ /3. O ECG mostrava fibrilação atrial, sobrecarga biventricular e extra-sístoles ventriculares. O ECO demonstrava dupla lesão mitral moderada com AE de 72 $\mathrm{mm}$. A conclusão do estudo hemodinâmico foi de dupla lesão mitral, insuficiência tricúspide e hipertensão pulmonar.

No dia 11.04 .83 , foi realizada troca de valva mitral por PBAP n: 26 e plástica de De Vega na tricúspide. No pós-operatório imediato evoluiu com hipotensão, sangramento, baixo débito grave e, apesar do tratamento instituído com aumento progressivo da dosagem de drogas inotrópicas, evoluiu para parada cardiorrespiratória irreversivel.

O estudo anatomopatológico revelou obstrução da via de saída do ventrículo esquerdo pela bioprótese implantada.

CASO 6 - Y. M. K., paciente do sexo feminino, raça branca, 36 anos de idade, com história reumática definida, tendo sido submetida a comissurutomia mitral há apenas um ano, tendo evoluído rapidamente para dupla lesão mitral com predominância de insuficiência e apresentando, também, insuficiência tricúspide. Apresentava dispnéia aos mínimos esforços nos últimos seis meses. Ao exame físico, na ausculta do foco mitral, havia $\mathrm{SS}++/ 3$ e SD $+/ 3$ e, no foco tricúspide, $S S+/ 3$ com fígado percutível e palpável a $6 \mathrm{~cm}$ do RCD. O ECG mostrava fibrilação atrial, sobrecarga ventricular esquerda e altera- 
POMERANTZEFF, P. M. A.; KIOKA, Y.; KAWABE, L. T.; AULER Jr., J. O. C.; LOPES, E. A.; VERGINELLI, G.; JATENE, A. D. - Obstrução da via de saída de ventrículo esquerdo por prótese mitral: relato de seis casos. Rev. Bras. Cir. Cardiovasc., 5(2): $120-124,1990$.

ção da repolarização ventricular. $O$ resultado do $E C O$ foi de dupla lesāo mitral com AE de $60 \mathrm{~mm}$ e DDVE de $55 \mathrm{~mm}$. O resultado do cateterismo cardiaco foi de dupla lesão mitral com predomínio de insuficiência tricúspide moderada.

A paciente foi submetida, no dia 28 de março de 1984, a substituição da valva mitral por bioprótese PBAP n: 26 e plástica de De Vega na tricúspide. No pós-operatório imediato, apresentou quadro de baixo débito importante, auscultando-se sopro sistólico intenso $(++/ 3)$ paraesternal esquerdo, principalmente no 3 : e 4: espaços intercostais esquerdos. Devido a essa evolução e ausculta, a paciente foi submetida a cateterismo cardíaco no pós-operatório imediato, estudo que demonstrou OVSVE pela bioprótese de pericárdio bovino e alto perfil. A pressão sistólica do ventrículo esquerdo baixo, isto é, da ponta do ventrículo esquerdo, foi de $220 \mathrm{~mm}$ $\mathrm{Hg}$ e a pressão sistólica medida na via de saída do ventrículo esquerdo foi de 123 , demonstrando-se, portanto, por esse estudo hemodinâmico, um gradiente sistólico entre a ponta e via de saída de ventrículo esquerdo de $97 \mathrm{~mm} \mathrm{Hg}$ (Figura 3).

Com o diagnóstico de OVSVE pela bioprótese de pericárdio de alto perfil, a paciente foi reoperada, substituindo-se a bioprótese de alto perfil por uma bioprótese porcina tipo Barone n: 20 de baixo perfil. A paciente apresentou boa evoluçāo pós-operatória, tendo sido reoperada em 1988, por rotura dessa bioprótese porcina.

\section{DISCUSSĀO}

Inicialmente, devemos ressaltar a importância do estudo anatomopatológico realizado rotineiramente em nossa instituição, que permitiu a constatação de ocorrência de casos de OVSVE na substituição mitral com biopróteses de PBAP.

$\mathrm{Na}$ literatura, encontramos relatos ${ }^{1,5 \cdot 7}$ de OVSVE ou arritmia fatal em pacientes submetidos a troca mitral

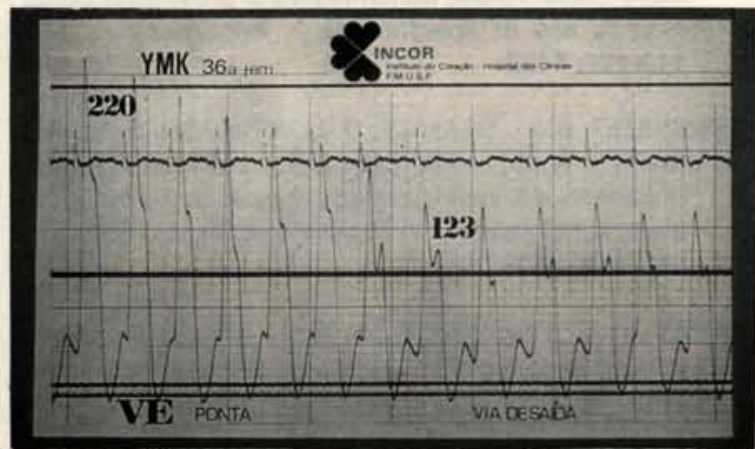

Fig. 3-Caso 6: Curva de pressão sistólica de ventriculo esquerdo demonstrando gradiente intraventricular entre a ponta e a via de saída de $97 \mathrm{mmHg}$ por prótese de bola de alto perfil ou biopróteses de alto perfil. Os autores, porém, só admitem tal ocorrência em pacientes com cavidade ventricular reduzida ou com hipertrofia ventricular esquerda.

Em quatro de nossos casos, mais especificamente nos casos 1, 2, 5 e 6, mesmo com a cavidade venticular esquerda dilatada, ocorreu OVSVE, sugerindo que nāo só a hipertrofia ou as dimensōes da cavidade são decisivas para determinar OVSVE.

JETT et alii ${ }^{3}$ demonstraram experimentalmente que, dependendo da posição em que se coloca a prótese, pode haver OVSVE pela haste, quando esta é de alto perfil. Colocando a haste direcionada na via de saída do ventriculo esquerdo, os autores demonstraram nítido angustiamento da mesma.

Os nossos casos de n: 2, 4 e 5 apresentavam grande dilatação do átrio esquerdo, com o anel mitral deslocado em direção ao septo interventricular, de maneira a modificar a posiçāo em que habitualmente são colocadas as biopróteses na substituição mitral. A modificação da anatomia, com variação do ângulo do anel mitral em relação ao septo interventricular também poderia favorecer a OVSVE. No caso de número 4 , havia traumatismo do septo interventriclar pela haste da bioprótese de alto perfil, demonstrando claramente a desproporçāo entre a anatomia do ventrículo esquerdo e o tamanho da prótese, e indicando, talvez, a causa da arritmia.

KAWAZOE et alii ${ }^{4}$, observando pacientes com grande átrio esquerdo submetidos a substituição da valva mitral que evoluíram em baixo débito e insuficiência respiratória, sugerem que deva ser feita plicatura do átrio esquerdo, no sentido de diminuir a compressão do ventrículo esquerdo, brônquio e parênquima pulmonar. Concordamos com essa opinião, pois a alteraçăo da geometria do ventrículo esquerdo pela compressão do átrio esquerdo poderia causar problemas na via de saída, nos casos de substituição mitral.

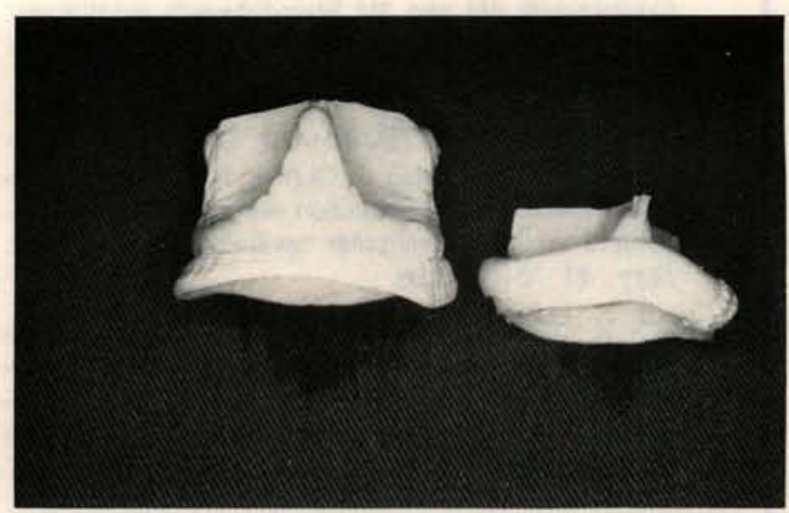

Fig. 4 - Biopróteses de pericárdio bovino modelo INCOR de alto e baixo perfil. 
POMERANTZEFF, P. M. A.; KIOKA, Y;; KAWABE, L. T.; AUlER Jr., J. O. C.; LOPES, E. A.; VERGINELLI, G.; JATENE, A. D. - Obstrução da via de saída de ventrículo esquerdo por prótese mitral: relato de seis casos. Rev. Bras. Cir. Cardiovasc., 5(2): 120-124, 1990.

Em cinco de nossos pacientes, ocorreu síndrome de baixo débito severo, sendo que a OVSVE foi diagnosticada apenas na necropsia. Todos estes pacientes receberam altas doses de medicação inotrópica no pós-operatório, sem que se obtivesse resposta adequada, o que pode ser explicado pelo aumento do gradiente entre a ponta do ventrículo esquerdo e a vida de saída do ventrículo esquerdo, levando a maior solicitação e desgaste do ventrículo esquerdo, com piora progressiva do quadro.

Esses dados são corroborados por JETT et alii ${ }^{2}$, que demonstraram haver aumento do gradiente intraventricular esquerdo em pacientes com ventrículo esquerdo pequeno submetidos a troca da mitral por prótese de Starr-Edwards ou biopróteses de lonescu-Shiley.

A OVSVE constatada nestes pacientes contribuiu, sem dúvida, para o desenvolvimento, no Serviço, de uma bioprótese de pericárdio bovino de baixo perfil, que passou a ser utilizada a partir de março de 1982 (Figura 4).

Em conclusão, acreditamos que o diagnóstico de OVSVE deva ser lembrado em pacientes submetidos a substituição da valva mitral que evoluem em baixo débito, principalmente se a cavidade ventricular esquerda for pequena ou hipertrófica, o átrio esquerdo muito grande, a posiçāo do anel mitral anômala ou, ainda, se for utilizada prótese de alto perfil.

Gostaríamos, ainda, de salientar que foi o estudo anatomopatológico de cinco casos de OVSVE que nos permitiu suspeitar de OVSV no pós-operatório imediato do caso de n: 6 , o que possibilitou, após confirmaçāo por cateterismo, a retroca de bioprótese com boa evolução do paciente.

RBCCV 44205-115

POMERANTZEFF, P. M. A.; KIOKA, Y.; KAWABE, L. T.; AULER Jr., J. O. C.; LOPES, E. A.; VERGINELLI, G.; JATENE, A. D. - Obstruction of the left ventricle outlet by mitral valve prosthesis: report of six cases. Rev. Bras. Cir. Cardiovasc., 5(2): 120-124, 1990.

ABSTRACT: From January/1982 to March/1984, 170 high profile bovine pericardium bioprostheses were implanted in the INCOR (Instituto do Coração), Hospital das Clínicas, Faculdade de Medicina da Universidade de São Paulo (FMUSP). Hospital mortality amongst these patients was $7.1 \%$. Anatomopathological studies identified five cases in which obstruction of the left ventricle outlet occurred after substitution of the mitral valve. Based on this experience, the indication of a hemodynamic study on the first postoperative day of a patient with an unexplained low cardiac output made it possible to identify obstruction of the left ventricle valve outlet in such patients, with resubstitution of the valve and a satisfactory evolution of the case. In this report we relate the six cases in detail and discuss the causes of the obstruction of the outlet in mitral valve substitutions.

DESCRIPTORS: left ventricle outlet, obstruction; heart valves, mitral, surgery, complications.

\section{REFERÊNCIAS BIBLIOGRÁFICAS}

1 GLANCY, D. L.; O'BRIEN, K. P.; REISS, R. L.; EPSTEIN, E. S.; MORROW, A. G. - Hemodynamic studies in patients with $2 \mathrm{M}$ and $3 \mathrm{M}$ Starr-Edwards prostheses: evidence of obstruction to left emptying. Circulation, 29 (Supl. 1): 90-113, 1969.

2 JETT, G. K.; JETT, M. D.; BARNHART, G. R.; VAN RIIJBASWITTEI, G. L.; JONES, J.; CLARK, R. E. - Lett ventricular outflow tract obsctruction with mitral valve replacement in small ventricular cavities. Ann. Thorac. Surg., 41: 70-74, 1986.

3 JETT, G. K.; JETT, M. D.; BOSCO, M. T. P.; VAN RİJBASWITTEI, G. L.; CLARK, R. E. - Left ventricular outflow tract obstruction following mitral valve replacement: effect of strut height and orientation. Ann. Thorac. Surg., 42: 299-303, 1986.

4 KAWAZOE, K.; FUJITA, T.; MANABE, H. - Surgical treatment of giant left atrium combined with mitral valvular disease. J. Thorac. Cardiovasc. Surg., 85: 885-892, 1983.

5 ROBERTS, W. C. - Complication of cardiac valve replacement: characteristic abnormalities of prostheses pertaining to any or specific site. $A m$. Heart J., 103: 113-122, 1982.

6 SHEPHERD, R. L.; GLANCY, D. L.; STINSON, E. B.; RO BERTS, W. C. - Hemodynamic confirmation of obstruction to left ventricular inflow by a caged-ball prosthetic mitral valve. J. Thorac. Cardiovasc. Surg., 65 : 252-254, 1979.

7 SPRAY, T. L. \& ROBERTS, W. C. - Structural changes in porcine xerografts: used as substitute cardiac valves. Am. J. Cardiol., 40: 319-330, 1977.

8 STARR, A. \& EDWARDS, M. L - Mitral replacement: clinical experience with a ball-valve prosthesis. Am. Surg., 154: 726-740, 1961. 